\title{
INVESTIGATION OF THE REPEATABILITY OF A CLEANING METHOD FOR SILICON SPHERES BY GRAVIMETRIC MEASUREMENTS
}

\author{
M. Mecke ${ }^{1}$, M. Borys ${ }^{2}$, E. Beyer ${ }^{3}$ \\ 1-2 Physikalisch-Technische Bundesanstalt (PTB), Bundesallee 100, 38116 Braunschweig, Germany \\ ${ }^{1}$ michael.mecke@ptb.de, ${ }^{2}$ michael.borys@ptb.de, \\ ${ }^{3}$ Physikalisch-Technische Bundesanstalt (PTB), Abbestraße 2-12, 10587 Berlin, Germany \\ edyta.beyer@ptb.de
}

\begin{abstract}
:
After a short description of the metrological relevance of silicon spheres and the applied cleaning method, mass measurements of silicon spheres in vacuum and the evaluation of the results are outlined. The results show that the mass of silicon spheres in vacuum stabilises in the microgram range within two to three days after cleaning and that the repeatability of the applied cleaning method can be characterised by a standard deviation in the order of two micrograms.
\end{abstract}

Keywords: New SI; kilogram; silicon spheres; cleaning method; repeatability; mass stability

\section{INTRODUCTION}

In the past, silicon spheres were used as primary density standards and for the determination of the Avogadro and the Planck constants with the smallest uncertainties [1], [2]. Since the introduction of the revised SI on 20 May 2019, the kilogram is no longer defined as the mass of the international prototype of the kilogram, but is instead derived from a fixed numerical value for the Planck constant [3]-[5]. One of the two independent primary methods which allow a realisation of the definition of the kilogram with relative uncertainties of a few parts in $10^{8}$ is the X-ray-crystal-density (XRCD) method. The silicon spheres which are currently used for the practical realisation of the definition of the mass unit are primary mass standards and are produced from nearly perfect isotopically enriched ${ }^{28} \mathrm{Si}$ crystals. Relevant influences on the mass of these spheres must be considered for the realisation, maintenance and dissemination of the mass unit [4]. Knowledge about their mass stability is required for uncertainty analysis, drift corrections and the determination of appropriate realisation intervals.

Complementary to ${ }^{28} \mathrm{Si}$ spheres, spheres produced from natural silicon $\left({ }^{\text {nat }} \mathrm{Si}\right)$ can be used for the maintenance and dissemination of the kilogram.
Since the mass stability of silicon spheres is, in addition to the growth of the oxide layer [6], influenced by the sorption of water and carbonaceous layers on the surface of the spheres, appropriate cleaning methods have been investigated and were successfully applied in the past [7]-[11]. In order to characterise the repeatability of the cleaning method used and to obtain reliable data for the assessment of its uncertainty contribution, extensive tests were performed at PTB in the past. The experimental design, results and conclusions are presented in the following sections.

\section{GRAVIMETRIC MEASUREMENTS}

Six test spheres with the identifiers Si15-12, SiSC01_a, SiSC01_b, SiSC01_c, SiSC01_d and SiSC01_e were used for the investigation of the repeatability of the cleaning method. This method starts with rinsing a sphere with distilled water, followed by a manual washing with a soap solution made of deconex OP 163 (2\%) and distilled water. The sphere is then placed under a stream of distilled water, rinsed with ethanol and finally dried with a cleaned microfibre cloth [11].

A silicon sphere with the identifier Sm14 was selected as a mass reference. The mass stability of this sphere is known from previous measurements and was checked in the course of the measurements by an additional reference sphere named Si12-06. The mass of the spheres was compared by means of a mass comparator, type Sartorius CCL1007, in a pressure range from $5 \times 10^{-5} \mathrm{hPa}$ to $5 \times 10^{-6} \mathrm{hPa}$. This mass comparator has a nominal load of $1 \mathrm{~kg}$, achieves a standard deviation in vacuum of less than $0.1 \mu \mathrm{g}$ and is equipped with a vacuum transfer system (see Figure 1), which allows the loading and unloading of the mass comparator without breaking the vacuum.

The mass measurements comprised two series, one in April/May 2017 with two to three cleanings and measurements of each sphere and a second one 
in September/October 2017 with four to five cleanings and measurements of each sphere. All the spheres were cleaned before the two measurement series and loaded into the mass comparator in air within two hours after completion of the cleaning procedure. The mass comparator was then evacuated and the mass comparisons were performed in vacuum over a period of three to fourteen days. After the measurements, the spheres were transferred under vacuum conditions (in a sealed container) to a surface analysis system, transferred to air by means of a vacuum transfer system, cleaned, loaded into the vacuum transfer system of the mass comparator again (within two hours after completion of the cleaning procedure), and were then transferred under vacuum into the mass comparator. After the completion of the first series and the beginning of the second, i.e. between the end of May and the beginning of September, all the test spheres were stored in their transport containers in air.

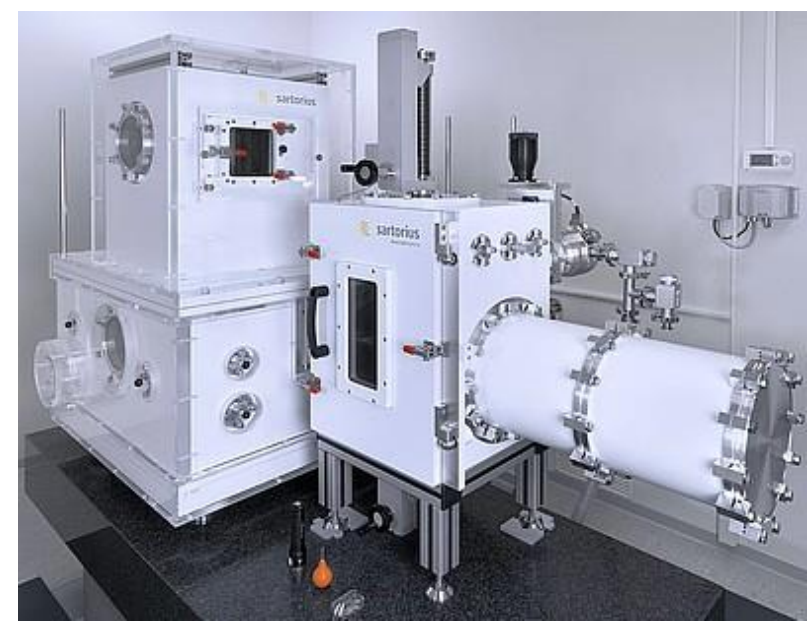

(a)

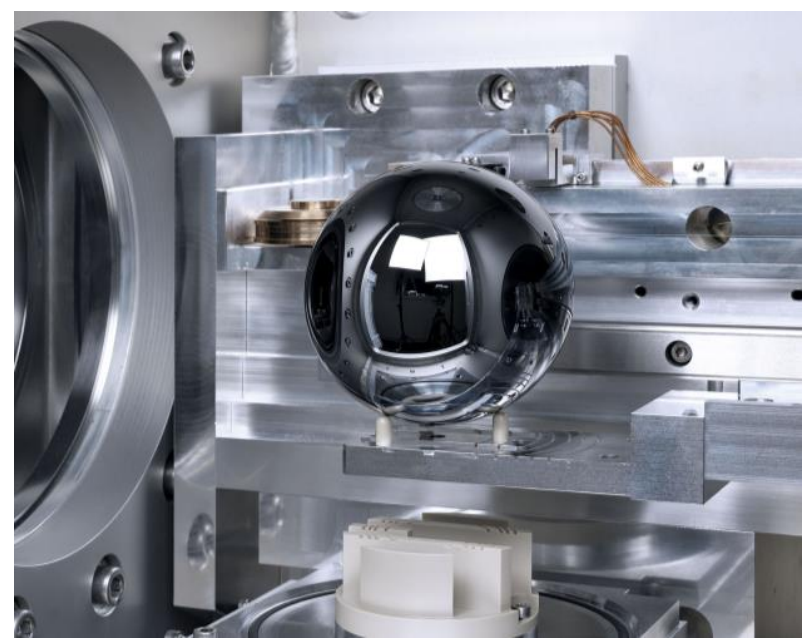

(b)

Figure 1: (a) Mass comparator used for the gravimetric measurements (type Sartorius CCL1007) in a pressure tight enclosure with a vacuum transfer system, (b) silicon sphere on the loading arm of the vacuum transfer system

\section{RESULTS AND DISCUSSION}

Each sphere was cleaned and measured between six and eight times (42 cleanings and mass measurements in total). Similar to the mass of kilogram prototypes, which are made of a platinumiridium alloy, and the mass of stainless steel weights, the mass of silicon spheres changes according to a non-linear function of time after applying a cleaning procedure with solvents. This non-linear behaviour can be described by the model function [12], [13]:

$$
m(t)=m_{\tau}\left(1-e^{-\frac{\left(t-t_{0}\right)}{\tau}}\right)+b\left(t-t_{0}\right)+m_{0}
$$

with the mass contribution of the non-linear term $m_{\tau}$, the time constant $\tau$, the slope of the linear term $b$ and the mass of the sphere $m_{0}$ at the time of the cleaning $t_{0}$. In order to get an estimate of the time constant $\tau$, least-squares adjustments of the parameters of the model equation (1) with measured data for the different test spheres were performed. Examples of the time dependence of the mass values of the test spheres are given in Figure 2. In order to allow a comparison of the different results in this figure, the data were shifted by the time of the cleaning $t_{0}$ and the mass of the sphere at the time of the cleaning $m_{0}$. As a result of the least-squares adjustment, the time constant $\tau$ was determined to be 1.3 days with a standard deviation of 0.5 days and a stability of the mass of the spheres in the microgram range two to three days after cleaning. Therefore, the results representing the mass of the spheres between two to three days after cleaning were chosen for the evaluation of the repeatability in order to ensure a good comparability, i.e. no corrections by means of equation (1) were applied. The results of the different spheres shown in Figure 2 cover a period of seven to fourteen days. In comparison to the linear term, the non-linear term of equation(1) dominates during this time. Therefore, an estimate of the slope $b$ of the linear term is not reliable and considered to be not significant regarding its influence on the mass difference measured two to three days after cleaning. However, for the slope $b$ a rough estimate of $35 \mathrm{ng} / \mathrm{d}$ with a standard deviation of $21 \mathrm{ng} / \mathrm{d}$ was determined from the measured data.

Before the first, between the first and the second and after the second series, the mass of the reference spheres Sm14 and Si12-06 in vacuum was determined traceable to the national prototype of the kilogram no. 52 in air. A mass stability within $\pm 0.7 \mu \mathrm{g}$ and $\pm 0.9 \mu \mathrm{g}$ was observed for the sphere Sm14 and Si12-06, respectively, with a standard uncertainty of $2.4 \mu \mathrm{g}$. The mass stability of the reference sphere Sm14 in the course of the measurements in vacuum was determined to be within $\pm 0.30(22) \mu \mathrm{g}$ in comparison to the second reference sphere Si12-06 for both, series 1 and 2. 
For each test sphere and each measurement, the mass difference to the mean value of the measured mass with respect to the reference sphere (Sm14) was determined and listed in Table 1. A diagram of the results is given in Figure 3. The curves illustrate the mass variation of the spheres after different cleanings. The observed difference between the minimum and the maximum deviation from the mean mass value of a single sphere ranges from $1.6 \mu \mathrm{g}$ to $4.9 \mu \mathrm{g}$. The maximum deviation from the mean value observed for all spheres and cleanings amounts to $3.0 \mu \mathrm{g}$. By means of a linear regression, a measure for the mass change of each test sphere from one cleaning to the next was estimated. The slope of the mass values observed for the different spheres varies between $0.26 \mu \mathrm{g}$ and $-0.72 \mu \mathrm{g}$ with a mean of $-0.25 \mu \mathrm{g}$ and a standard deviation of $0.32 \mu \mathrm{g}$.

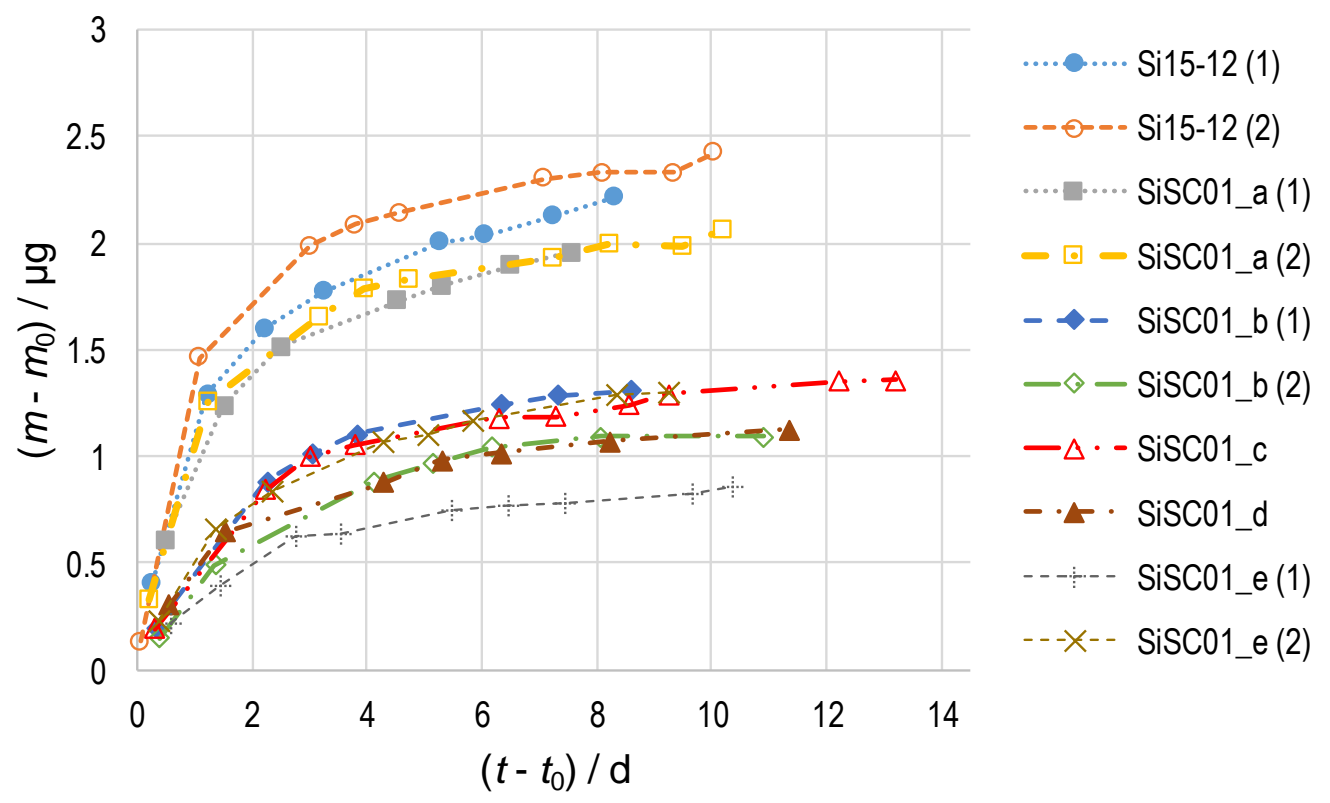

Figure 2: Examples of the measured time dependence of the mass of the test spheres in vacuum after applying the cleaning procedure in air at the time $t=t_{0}$. All spheres were cleaned before the measurements and loaded into the mass comparator in air within two hours after completion of the cleaning procedure. The last figure in parentheses behind the identifier of the sphere in the legend indicates the number of the measurement in the case that more than one curve is shown for a sphere.

Table 1: Results of the measured mass differences between the test spheres and the reference sphere (Sm14). In order to allow a better comparability, the individual mass differences of a sphere are given with respect to their observed mean value $(\bar{m})$ and the time since their first cleaning.

\begin{tabular}{|c|c|c|c|c|c|c|c|c|c|c|c|}
\hline \multicolumn{2}{|c|}{ Si15-12 } & \multicolumn{2}{c|}{ SiSC01_a } & \multicolumn{2}{c|}{ SiSC01_b } & \multicolumn{2}{c|}{ SiSC01_c } & \multicolumn{2}{c|}{ SiSC01_d } & \multicolumn{2}{c|}{ SiSC01_e } \\
\hline $\begin{array}{c}\text { Time } \\
\text { in d }\end{array}$ & $\begin{array}{c}\left(\boldsymbol{m}_{\boldsymbol{i}}-\overline{\boldsymbol{m}}\right) \\
\text { in } \boldsymbol{\mu g}\end{array}$ & $\begin{array}{c}\text { Time } \\
\text { in d }\end{array}$ & $\begin{array}{c}\left(\boldsymbol{m}_{\boldsymbol{i}}-\overline{\boldsymbol{m}}\right) \\
\text { in } \boldsymbol{\mu g}\end{array}$ & $\begin{array}{c}\text { Time } \\
\text { in d }\end{array}$ & $\begin{array}{c}\left(\boldsymbol{m}_{\boldsymbol{i}}-\overline{\boldsymbol{m}}\right) \\
\text { in } \boldsymbol{\mu g}\end{array}$ & $\begin{array}{c}\text { Time } \\
\text { in d }\end{array}$ & $\begin{array}{c}\left(\boldsymbol{m}_{\boldsymbol{i}}-\overline{\boldsymbol{m}}\right) \\
\text { in } \boldsymbol{\mu g}\end{array}$ & $\begin{array}{c}\text { Time } \\
\text { in d }\end{array}$ & $\begin{array}{c}\left(\boldsymbol{m}_{\boldsymbol{i}}-\overline{\boldsymbol{m}}\right) \\
\text { in } \boldsymbol{\mu g}\end{array}$ & $\begin{array}{c}\text { Time } \\
\text { in d }\end{array}$ & $\begin{array}{c}\left(\boldsymbol{m}_{\boldsymbol{i}}-\overline{\boldsymbol{m}}\right) \\
\text { in } \boldsymbol{\mu g}\end{array}$ \\
\hline 2.3 & 1.15 & 2.4 & 1.80 & 2.4 & -0.44 & 1.7 & 0.67 & 1.8 & 0.44 & 2.6 & -0.94 \\
\hline 9.1 & -0.60 & 15.5 & 3.04 & 15.6 & 3.03 & 15.7 & 1.19 & 15.8 & -0.21 & 11.9 & 0.15 \\
\hline 21.3 & 0.15 & 21.4 & -0.26 & 23.5 & 0.05 & 24.7 & 0.94 & 134.0 & -0.12 & 128.0 & -0.36 \\
\hline 134.4 & 0.63 & 134.5 & -0.71 & 134.6 & -0.60 & 133.9 & 0.08 & 145.7 & 0.99 & 140.8 & 0.45 \\
\hline 140.2 & 0.17 & 141.5 & -0.55 & 142.3 & -0.81 & 137.6 & 0.21 & 153.8 & -0.63 & 147.9 & -0.22 \\
\hline 148.4 & 0.28 & 149.2 & -1.41 & 151.4 & -0.20 & 143.7 & -0.57 & 165.7 & -0.48 & 161.8 & 0.92 \\
\hline 156.1 & -0.40 & 156.2 & -1.90 & 157.1 & -1.04 & 152.6 & -1.07 & & & & \\
\hline 168.2 & -1.39 & & & & & 156.4 & -1.46 & & & & \\
\hline
\end{tabular}




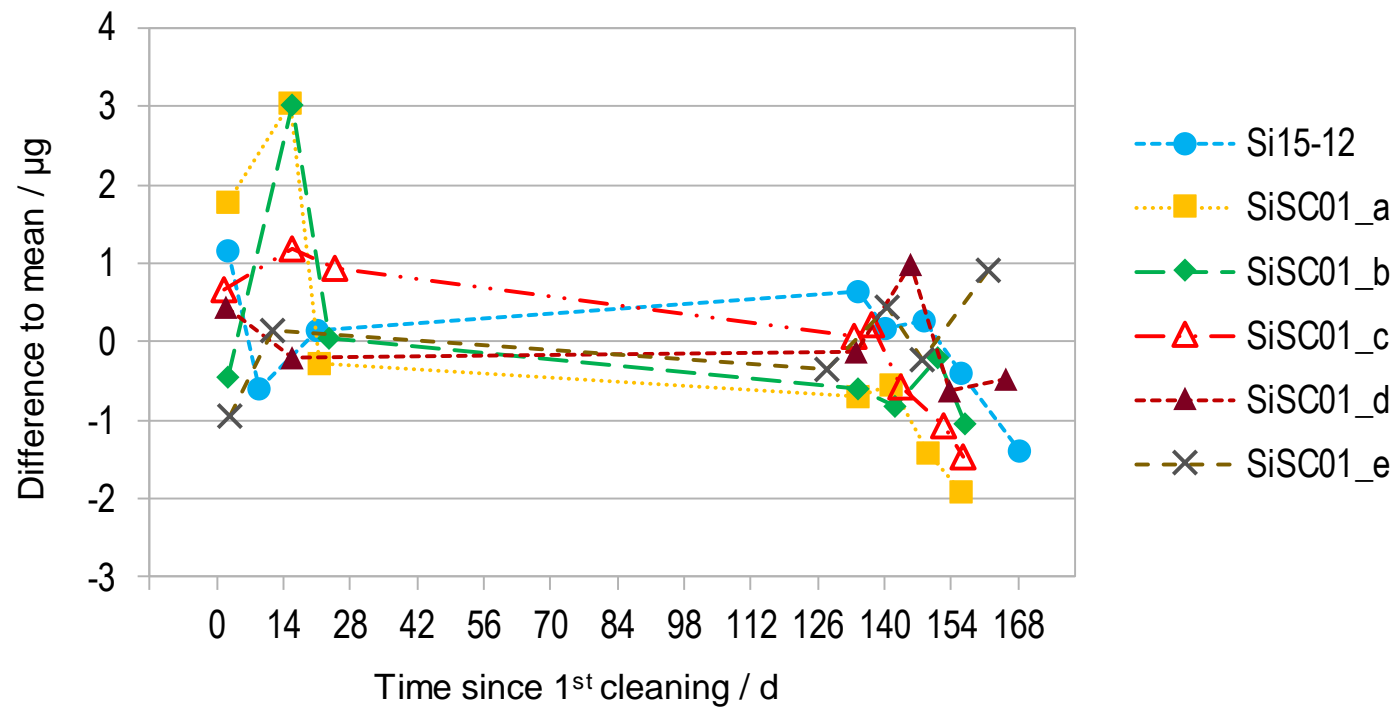

Figure 3: Diagram of the data given in Table 1 for the measured mass differences between the test spheres and the reference sphere (Sm14). The individual mass differences of a sphere are given with respect to their observed mean value $(\bar{m})$ as a function of the time since the first cleaning.

Table 2: Standard deviation of the observed mass variations after cleaning given for the individual test spheres and as a pooled standard deviation of all measurements.

\begin{tabular}{|c|c|c|c|c|c|c|}
\hline \multicolumn{5}{|c|}{$\begin{array}{c}\text { Standard deviation } \\
\text { in } \boldsymbol{\mu g}\end{array}$} & $\begin{array}{c}\text { Pooled standard } \\
\text { deviation } \\
\text { in } \boldsymbol{\mu g}\end{array}$ \\
\hline Si15-12 & SiSC01_a & SiSC01_b & SiSC01_c & SiSC01_d & SiSC01_e & \\
\hline 0.8 & 1.8 & 1.4 & 1.0 & 0.6 & 0.7 & 1.1 \\
\hline
\end{tabular}

The difference between the mean mass values of series 1 and series 2 amounts to $0.9 \mu \mathrm{g}$, which may be caused by a mass change of the reference sphere due to the second cleaning. Since this value is in the order of magnitude of the uncertainties of the measured mass differences, which are estimated to be about one microgram (standard uncertainty $(k=1)$, influence of the repeatability of loading and unloading considered, but without influence of the cleaning process), this is not considered to be a proof. Therefore, a correction based on the mass difference between mean values of the series was not applied.

In order to provide a measure for the mass variations after the cleanings, the standard deviation of the results was calculated for the individual test spheres and for all the measurements (see Table 2). The observed standard deviations are smaller than two micrograms, i.e. the standard deviations are in the order of magnitude of the uncertainties of the measured mass differences.

\section{SUMMARY AND CONCLUSIONS}

The cleaning method applied at PTB for the cleaning of silicon spheres was investigated by gravimetric measurements in vacuum. The results show that the mass of silicon spheres in vacuum changes according to a non-linear function of time after applying the cleaning procedure in air. The non-linear behaviour was described by a non-linear model function. Based on a least-squares adjustment, the time constant of this function was estimated to be 1.3 days with a standard deviation of 0.5 days. The stability of the mass of the spheres was observed to be in the microgram range two to three days after cleaning. The repeatability of the cleaning method applied at PTB can be characterised by a standard deviation in the order of two micrograms. It can be concluded that this uncertainty contribution is not significant in comparison to the present uncertainty of the realisation of the new definition of the kilogram by means of silicon spheres.

\section{ACKNOWLEDGEMENTS}

The authors would like to thank all their colleagues who were involved in the manufacture of the spheres in the workshop for ultra-precision machining at PTB. 


\section{REFERENCES}

[1] B. Andreas et al., "Determination of the Avogadro Constant by Counting the Atoms in a ${ }^{28} \mathrm{Si}$ Crystal", Phys. Rev. Lett., vol. 106, 030801-1-4, 2011.

[2] P. J. Mohr, D. B. Newell, B. N. Taylor, E. Tiesinga, "Data and analysis for the CODATA 2017 special fundamental constants adjustment", Metrologia, vol. 55, pp. 125-146, 2018.

[3] Resolutions of the $26^{\text {th }}$ meeting of the General Conference of Weights and Measures (CGPM), Versailles, 13-16 November 2018. Online [accessed 20201029]:

https://www.bipm.org/utils/common/pdf/CGPM2018/26th-CGPM-Resolutions.pdf

[4] Mise en pratique for the definition of the kilogram in the new SI. Online [accessed 20201029]: https://www.bipm.org/utils/en/pdf/si-mep/SIApp2-kilogram.pdf

[5] Bureau International des Poids et Mesures: The International System of Units (SI), $9^{\text {th }}$ SI Brochure, 2019. Online [accessed 20201029]:

https://www.bipm.org/utils/common/pdf/sibrochure/SI-Brochure-9-EN.pdf

[6] M. Borys, M. Mecke, U. Kuetgens, I. Busch, M. Krumrey, P. Fuchs, K. Marti, H. Bettin, "The growth of the oxide layer on silicon spheres and its influence on their mass stability", Proc. Joint IMEKO International TC3, TC5 and TC22 Conference, Cape Town, Republic of South Africa, 6 pages, 2014.
[7] M. P. Seah, S. J. Spencer, "Ultrathin $\mathrm{SiO}_{2}$ on Si. I. Quantifying and removing carbonaceous contamination", J. Vac. Sci. Technol. A, vol. 21, no. 2, pp. 345-352, March/April 2003.

[8] H. Bettin, D. Schiel, M. Vogtmann, H. Niemann, "Cleaning of silicon density standards", in Proc. XIX IMEKO World Congress, pp. 179-181, 2009.

[9] A. Picard, P. Barat, M. Borys, M. Firlus, S. Mizushima, "State-of-the-art mass determination of ${ }^{28} \mathrm{Si}$ spheres for the Avogadro project", Metrologia, vol. 48, pp. S112-S119, 2011.

[10] N. Kuramoto, K. Fujii, A. Nicolaus, G. Bartl, M. Gray, P. Manson, W. Giardini, "Diameter Comparison of a Silicon Sphere for the International Avogadro Coordination Project", IEEE Transactions on Instrumentation and Measurement, vol. 60, no. 7, pp. 2615-2620, July 2011

[11] J. W. Chung, V. Görlitz, M. Vogtmann, E. Beyer, F. Härtig, "The PTB Cleaning Procedure for Silicon Spheres", Physikalisch-Technische Bundesanstalt, Braunschweig, 2016. Online [accessed 20201029]:

https://www.ptb.de/cms/ptb/fachabteilungen/abt1/f b-11/si-kg.html

[12] M. Gläser, "Time dependence of the mass of kilogram prototypes after cleaning", personal communication, Braunschweig, 2003.

[13] K. Marti, P. Fuchs, S. Russi, "Traceability of mass II: a study of procedures and materials", Metrologia, vol. 52 pp. 89-103, 2015. 